\title{
Correction to: Use of Urban Health Indicator Tools by Built Environment Policy- and Decision-Makers: a Systematic Review and Narrative Synthesis
}

\author{
Helen Pineo 1 - Ketevan Glonti • Harry Rutter • \\ Nici Zimmermann • Paul Wilkinson • Michael Davies
}

Published online: 26 December 2019

(C) The New York Academy of Medicine 2019

\section{Correction to: J Urban Health \\ https://doi.org/10.1007/s11524-019-00378-w}

There were errors in this article as originally published. The following corrections to citations of references should be noted:

- Tables 1 and 2 citation [9] should refer to reference 40 Shepherd and McMahon (2009)

- Tables 1 and 2 citation [6] should refer to appendix reference 6 Landis and Sawicki (1988) (note, this reference is missing from the main list of references)

The online version of the original article can be found at https://oi. org/10.1007/s11524-019-00378-w

H. Pineo $(\bowtie) \cdot$ N. Zimmermann $\cdot$ M. Davies Institute for Environmental Design and Engineering, Bartlett School of Environment, Energy and Resources, University College London, Central House, 14 Upper Woburn Place, London WC1H 0NN, UK

e-mail: helen.pineo@ucl.ac.uk

K. Glonti

School of Humanities and Social Sciences, University of Split, Split, Croatia

K. Glonti

INSERM, U1153 Epidemiology and Biostatistics Sorbonne Paris Cité Research Center (CRESS), Methods of Therapeutic Evaluation of Chronic Diseases Team (METHODS), 75014 Paris, France
- Tables 1 and 2 citation [8] should refer to appendix reference 8 Lowe et al. (2015) (note, this reference is missing from the main list of references)

- Tables 1 and 2 citation [10] should refer to reference 36 Van Assche et al. (2010)

- Tables 1 and 2 citation [7] should refer to reference 42 Lerman (2011)

- Table 2 citation [5] should refer to reference 39 Hunt and Lewin (2000)

K. Glonti

Paris Descartes University, Sorbonne Paris Cité, Paris, France

H. Rutter

Department of Social \& Policy Sciences, University of Bath, Claverton Down, Bath, UK

P. Wilkinson

Department of Social and Environmental Health Research, London School of Hygiene and Tropical Medicine, Keppel Street, London WC1E 7HT, UK 
- Table 2 citation [1] should refer to reference 34 Bhatia (2014)

- Table 2 citation [4] should refer to reference 35 Farhang et al. (2008)

- Table 2 citation [2] should refer to reference 25 Corburn and Cohen (2012) (note the mistake is made twice in Table 2)

- Table 2 citation [3] should refer to reference 41 Corburn et al. (2014)

- Appendix Table 6 citation 12 (note these are not currently referenced in the same way as the rest of the paper, e.g. in the same style, number order and with hyperlinks) should refer to reference 20 Sterman (2000) (note this reference occurs in rows 1, 3, 4, 5, 6)

- Appendix Table 6 citation 2 should refer to reference 25 Corburn and Cohen (2012) (note this reference occurs in row 1 and the final column in all 4 bullets)

- Appendix Table 6 citation 13 should refer to reference 5 McCarney and McGahan (2015) (note this reference occurs in rows 1 and 3 )

- Appendix Table 6 citation 14 refers to appendix reference 14 Glouberman et al. (2006) and should be placed in the main reference body and renumbered to avoid confusion with other citations (note this reference occurs in rows 2 and 7)
- Appendix Table 6 citation 15 refers to reference 21 Luke and Stamatakis (2012) (note this reference occurs in rows 2 and 7)

- Appendix Table 6 citation 16 refers to appendix reference 16 Block et al. (2008) and should be placed in the main reference body and renumbered to avoid confusion with other citations (note this reference occurs in row 2).

- Appendix Table 6 citation 17 refers to appendix reference 17 AARP (n.d.) and should be placed in the main reference body and renumbered to avoid confusion with other citations (note this reference occurs in row 2).

- Appendix Table 6 citation 9 should refers to reference 40 Shepherd and McMahon (2009) (note this reference occurs the final column, bullet 3 )

- Appendix Table 6 citation 10 should refer to reference 36 Van Assche et al. (2010) (note this reference occurs the final column, bullets 3 and 4 )

The original article has been corrected.

Publisher's Note Springer Nature remains neutral with regard to jurisdictional claims in published maps and institutional affiliations. 